\title{
PELABELAN TOTAL SISI ANTI AJAIB SUPER (PTSAAS) PADA GABUNGAN GRAF BINTANG GANDA DAN LINTASAN
}

\author{
Nurainun $^{1}$, S. Musdalifah ${ }^{2}$, I. W. Sudarsana ${ }^{3}$ \\ 1,2,3Jurusan Matematika FMIPA Universitas Tadulako, \\ Jalan sukarno-Hatta Palu, \\ 1nununk_nice16@yahoo.co.id, 2selvymusdalifah@yahoo.com, ${ }^{3}$ sudarsanaiwayan@yahoo.co.id
}

\begin{abstract}
Abstrak
Pelabelan Total (a,d) sisi anti ajaib (a,d)- TSAA pada graf $G(V, E)$ adalah pemetaan satu-satu $V(G) \cup E(G)$ pada $\{1,2 \ldots, p+q\}$, sedemikian sehingga himpunan bobot sisi dari semua sisi di $\mathrm{G}$ adalah $\{a, a+d, a+2 d, \ldots . a+$ $(e-1) d\}$ sama dengan $W\{f(u)+f(u v)+f(v): u v \in E(G)\}$ untuk suatu bilangan bulat positif $a>0$ dan $d \geq 0$. Dimana , $p=$ banyaknya titik dan $q=$ banyaknya sisi. Pelabelan $\lambda$ dikatakan pelabelan total sisi ajaib super (TSAAS) jika mempunyai sifat bahwa setiap titik memperoleh label terkecil, $\lambda(V)=\{1,2, \ldots, p\}$ sebuah graf $G(V, E)$ dikatakan TSAA (TSAAS) jka terdapat pelabelan TSA (TSAAS) pada graf tersebut, berbeda dua. Pada penelitian ini, akan ditunjukkan bahwa gabungan dua graf dari dua graf lintasan dan sebuah graf bintang ganda atau dua graf bintang ganda dan sebuah graf lintasan adalah TSAAS dengan pelabelan $(a, d)=\left(\frac{9 n}{2}+5,2\right)$ untuk $n \geq 4$ genap, $(a, d)=\left(\frac{9 n+3}{2}+5,2\right)$ untuk $n \geq 5$ ganjil, $(a, d)=\left(\frac{9 n}{2}+6,2\right)$ untuk $n \geq 4$ genap, $(a, d)=\left(\frac{9 n+}{2}+5,2\right)$ untuk $n \geq 4$ ganjil.
\end{abstract}

\section{Kata Kunci : : Bintang Ganda, Lintasan, TSAA, TSAAS}

\section{Abstract}

Total Labeling $(a, d)$ the anti- magic edge, $(a, d)-T S A A$ in a graph $G(V, E)$ is one to one mapping from $V(G) \cup$ $E(G)$ to $\{1,2 \ldots, p+q\}$, such that the set of weight of all edges in the $\mathrm{G}$ is $\{a, a+d, a+2 d, \ldots . a+(e-1) d\}$ is equal to $W\{f(u)+f(u v)+f(v): u v \in E(G)\}$ for a positive integer $a>0$ and $d \geq 0$. Where $\mathrm{p}=$ the member of points and $q=$ the member of side. Labeling of $\lambda$ is called anti magic total super labeling (TSAAS) if it $\lambda(V)=$ $\{1,2, \ldots, p\}$ a graph $G(V, E)$ is called TSAA (TSAAS) if there TSA labeling (TSAAS) on the graph labeling, unlike the two. In this research, will be shown that the combine of two graphs from two graphs track and a double star graph or two double star graph and a graph track is TSAAS with labeling $(a, d)=\left(\frac{9 n}{2}+5,2\right)$ for $n \geq 4$ even, $(a, d)=\left(\frac{9 n+3}{2}+5,2\right)$ for $n \geq 5$ uneven,,$(a, d)=\left(\frac{9 n}{2}+6,2\right)$ for $n \geq 4$ even, $(a, d)=\left(\frac{9 n+}{2}+5,2\right)$ for $n \geq 4$ uneven.

\section{Keyword $\quad$ : Double Stars, Track, TSAA, TSAAS}




\section{Pendahuluan}

\subsection{Latar Belakang}

Matematika merupakan salah satu ilmu yang banyak dimanfaatkan untuk menyelesaikan permasalahan di berbagai bidang. Salah satu cabang ilmu matematika yang sering digunakanuntuk menyelesaikan suatu permasalahan adalah teori graf.

Walaupun graf telah banyak dipelajari, namun semakin majunya tekhnologi komputer, telah membangkitkan minat baru untuk mempelajari graf dan menjadikan graf sebagai salah satu cabang matematika yang akhir-akhir ini berkembang pesat. Diantaranya adalah banyaknya penemuanpenemuan baru mengenai graf. Mulai jenis-jenis graf, macam-macam pelabelannya dan cara melabelkannya. Teori graf mulai dikenal pada saat seorang matematikawan berkebangsaan Swiss, bernama Leonhard Euler, berhasil mengungkapkan Misteri Jembatan Konigsberg pada tahun 1736. Di Kota Konigsberg (sekarang bernama Kalilingrad, di Uni Soviet) mengalir sebuah sungai bernama sungai Pregel. Di tengah sungai tersebut terdapat dua buah pulau. Dari kedua pulau tersebut terdapat jembatan yang menghubungi ke tepian sungai dan diantara kedua pulau.

Graf digunakan untuk merepresentasikan objek-objek diskrit dan hubungan antara objekobjek tersebut. Representasi visual dari graf adalah dengan menyatakan objek dengan simpul, noktah, bulatan, titik, atau vertex, sedangkan hubungan antara objek dinyatakan dengan garis atau edge. Salah satu aplikasi yang berkaitan dengan pelabelan graf adalah salah satunya dengan menggunakan sistem kriptografi diantaranya sebagai penguncian pesan.

Pelabelan graf merupakan suatu topik dalam teori graf. Objek kajiannya berupa graf yang secara umum direpresentasikan oleh titik dan sisi serta himpunan bagian bilangan cacah yang disebut label. Pertama kali diperkenalkan oleh Sadlack (1964), kemudian Stewart (1966), Kotzig dan Rosa (1970) (dalam Gallian, 2011).

Pelabelan suatu graf adalah suatu pemetaan dari himpunan elemen graf (vertex, edge, atau vertex dan edge) terhadap bilangan bulat positif. Pelabelan graf adalah pemberian label pada elemen-elemen tertentu dari graf tersebut dengan menggunakan bilangan bulat positif. Elemenelemen graf itu sendiri meliputi himpunan titik, himpunan sisi, dan himpunan titik dari sisi.

Pelabelan titik adalah pelabelan graf dimana domainnya merupakan himpunan titik. Pelabelan sisi adalah pelabelan graf dimana domainnyamerupakan himpunan sisi, sedangkan pelabelan total adalah pelabelanyang domainnya merupakan gabungan dari himpunan titik dan sisi. Terdapat beberapa jenis pelabelan graf, diantaranya adalah pelabelan gracefull, pelabelan harmoni, pelabelan 
total tak beraturan, pelabelan ajaib, dan pelabelan anti ajaib. Dalam pelabelan ajaib terdapat beberapa macam pelabelan, diantaranya adalah pelabelan total titik ajaib, pelabelan total sisi ajaib, pelabelan total titik ajaib superdan pelabelan total sisi ajaib super, sedangkan pada pelabelan anti ajaib terdapat pelabelan total titik anti ajaib dan pelabelan total sisi anti ajaib. Pada pelabelan total sisi anti ajaib terbagi lagi ada yang super dan ada yang tidak.Dalam hal ini, penelitian ini fokus pada Pelabelan Total Sisi Anti Ajaib Super.

Dalam penelitian sebelumnya sudah ada beberapa peneliti yang telah mengerjakan materi Pelabelan Total Sisi Anti Ajaib Super diantaranya "Eva Solina, pelabelan total (a,d)-sisi-anti ajaib super pada graf $C_{n} \cup K_{2}$ " dan "Sri Mei Itasari (2011), pelabelan total (a,d) $C_{4}$ anti ajaib super pada graf prisma $C_{n} \times P_{2}$ "dan masih banyak lagi paper-paper yang terdapat di Gallian (2011).

Dalam penelitian ini yang diangkat adalah gabungan graf bintang ganda dan graf lintasan karena belum ada yang mengerjakannya, ini masih menjadi masalah yang terbuka yang didasari oleh hasil survey dari paper Gallian (2011).

\section{Metode Penelitian}

\subsection{Lokasi dan Tempat Penelitian}

Lokasi dan tempat penelitian bertempat di Jurusan Matematika Fakultas MIPA Universitas Tadulako.

\subsection{Alat dan Bahan}

Adapun alat dan bahan yang digunakan pada penelitian ini adalah sebuah alat tulis menulis dan laptop dengan menggunakan software pemrograman Microsoft Office Visio 2003.

\subsection{Jenis dan sumber data}

Jenis data yang digunakan pada penelitian ini adalah data kuantitatif yaitu data yang berupa angka. Sedangkan sumber data yang digunakan dalam penelitian ini adalah data sekunder.

\subsection{Teknik Analisa Data}

Teknik yang digunakan adalah studi literatur, yaitu mengumpulkan informasi dari beberapa buku, artikel dan jurnal yang berkaitan dengan Pelabelan TSAAS.

\subsection{Prosedur Penelitian}

Penelitian dapat dilakukan sesuai dengan prosedur dibawah ini :

1. Memulai penelitian 
2. Menotasikan titik dan sisi pada2 $\mathrm{P}_{n} \cup S_{\frac{n}{2}+1}, \frac{n}{2}-1$ untuk $n \geq 4$ genap dan $2 \mathrm{P}_{n} \cup S_{\frac{n+3}{2}}, \frac{n-3}{2}$ untuk $n \geq 5$ ganjil. Serta $\mathrm{P}_{n} \cup 2 S_{\frac{n}{2}+1}, \frac{n}{2}-1$ untuk $n \geq 4$ genap dan $\mathrm{P}_{n} \cup 2 S_{\frac{n+3}{2}}, \frac{n-3}{2}$ untuk $n \geq 5$ ganjil.

3. Memberikan label untuk titik dan sisi pada $2 \mathrm{P}_{n} \cup S_{\frac{n}{2}+1}, \frac{n}{2}-1$ untuk $n \geq 4$ genap dan $2 \mathrm{P}_{n} \cup$ $S_{\frac{n+3}{2}}, \frac{n-3}{2}$ untuk $n \geq 5$ ganjil. Serta $\mathrm{P}_{n} \cup 2 S_{\frac{n}{2}+1}, \frac{n}{2}-1$ untuk $n \geq 4$ genap dan $\mathrm{P}_{n} \cup 2 S_{\frac{n+3}{2}}, \frac{n-3}{2}$ untuk $n \geq 5$ ganjil.

4. Membuat formula Pelabelan TSAAS pada $2 \mathrm{P}_{n} \cup S_{\frac{n}{2}+1}, \frac{n}{2}-1$ untuk $n \geq 4$ genap dan $2 \mathrm{P}_{n} \cup$ $S_{\frac{n+3}{2}}, \frac{n-3}{2}$ untuk $n \geq 5$ ganjil. Serta $\mathrm{P}_{n} \cup 2 S_{\frac{n}{2}+1}, \frac{n}{2}-1$ untuk $n \geq 4$ genap dan $\mathrm{P}_{n} \cup 2 S_{\frac{n+3}{2}}, \frac{n-3}{2}$ untuk $n \geq 5$ ganjil.

5. Membangkitkan konstanta anti ajaib pada $2 \mathrm{P}_{n} \cup S_{\frac{n}{2}+1}, \frac{n}{2}-1$ untuk $n \geq 4$ genap dan $2 \mathrm{P}_{n} \cup$ $S_{\frac{n+3}{2}}, \frac{n-3}{2}$ untuk $n \geq 5$ ganjil. Serta $\mathrm{P}_{n} \cup 2 S_{\frac{n}{2}+1}, \frac{n}{2}-1$ untuk $n \geq 4$ genap dan $\mathrm{P}_{n} \cup 2 S_{\frac{n+3}{2}}, \frac{n-3}{2}$ untuk $n \geq 5$ ganjil.

6. Hasil

7. Selesai

III. Hasil dan Pembahasan

3.1. Pelabelan TSAAS Pada Graf $2 P_{n} \cup S_{\frac{n}{2}+1}, \frac{n}{2}-1$ untuk $n \geq 4$ genap Graf $2 \mathrm{P}_{n} \cup \mathrm{S}_{\frac{n}{2}+1}, \frac{n}{2}-1$ untuk $n \geq 4$ genap, dapat digambarkan sebagai berikut :
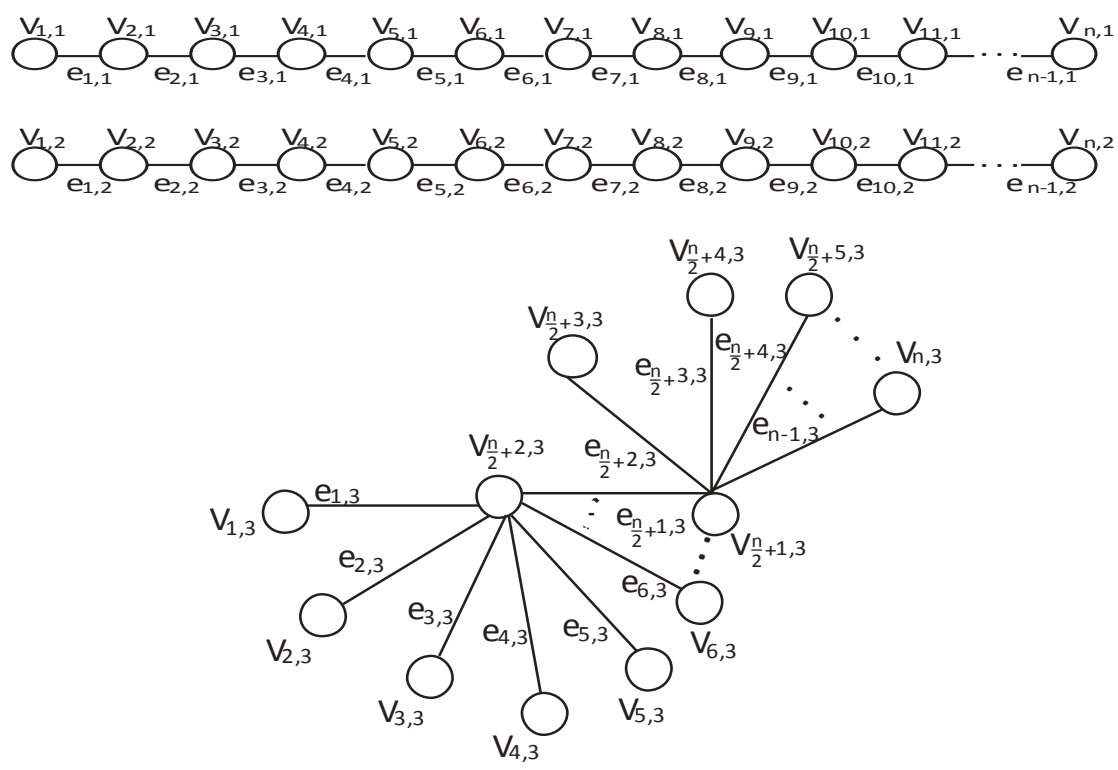

Gambar 1: Penotasian Titik dan Sisi Graf $2 \mathrm{P}_{n} \cup \mathrm{S}_{\frac{n}{2}+1}, \frac{n}{2}-1$ 
Berdasarkan gambar diatas, dapat dinotasikan graf $2 \mathrm{P}_{n} \cup \mathrm{S}_{\frac{n}{2}+1}, \frac{n}{2}-1$ dengan himpunan titik dan sisinya sebagai berikut :

$\mathrm{V}\left(2 \mathrm{P}_{n} \cup \mathrm{S}_{\frac{n}{2}+1}, \frac{n}{2}-1\right)=\left\{v_{i, j} \mid 1 \leq j \leq 3,1 \leq i \leq n\right\}$

$\mathrm{E}\left(2 \mathrm{P}_{n} \cup \mathrm{S}_{\frac{n}{2}+1}, \frac{n}{2}-1\right)=\left\{e_{i, j} \mid 1 \leq j \leq 3,1 \leq i \leq n-1\right\}$,

dimana

$e_{i, 1}=v_{i, 1} v_{i+1,1}, 1 \leq i \leq n-1$

$e_{i, 2}=v_{i, 2} v_{i+1,2}, 1 \leq i \leq n-1$

$e_{i, 3}=\left\{\begin{array}{c}v_{i, 3} v_{\frac{n}{2}+2,2}, 1 \leq i \leq \frac{n}{2}+1 \\ v_{\frac{n}{2}+1,2} v_{i+1,2}, \frac{n}{2}+2 \leq i \leq n-1\end{array}\right.$

3.1.1. Untuk $n=4 \rightarrow 2 \mathrm{P}_{4} \cup \mathrm{S}_{3,1}$

Pelabelan TSAAS pada graf $2 \mathrm{P}_{4} \cup \mathrm{S}_{3,1}$, dapat dilakukan seperti pada gambar dibawah ini :

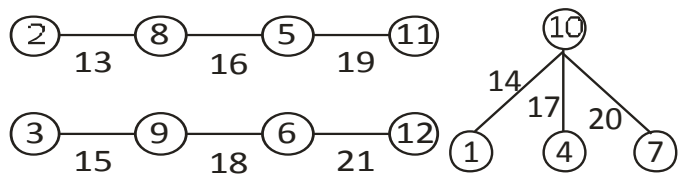

Gambar 2 : Pelabelan Titik dan Sisi Graf $2 \mathrm{P}_{4} \cup \mathrm{S}_{3,1}$

Perhatikan bahwa titik diberi label dengan anggota himpunan $\lambda(\mathrm{V})=\{1,2, \ldots, 12\}$ dan banyaknya titik $n=4$. Jika $\lambda$ adalah pelabelan TSAAS untuk graf $2 \mathrm{P}_{4} \cup \mathrm{S}_{3,1}$ maka diperoleh :

$\begin{array}{lll}\lambda\left(\mathrm{v}_{1,1}\right)=2 & \lambda\left(\mathrm{v}_{1,2}\right)=3 & \lambda\left(\mathrm{v}_{1,3}\right)=1 \\ \lambda\left(\mathrm{v}_{2,1}\right)=8 & \lambda\left(\mathrm{v}_{2,2}\right)=9 & \lambda\left(\mathrm{v}_{2,3}\right)=2 \\ \lambda\left(\mathrm{v}_{3,1}\right)=5 & \lambda\left(\mathrm{v}_{3,2}\right)=6 & \lambda\left(\mathrm{v}_{3,3}\right)=7 \\ \lambda\left(\mathrm{v}_{4,1}\right)=11 & \lambda\left(\mathrm{v}_{4,2}\right)=12 & \lambda\left(\mathrm{v}_{4,3}\right)=10 \\ \lambda\left(\mathrm{e}_{1,1}\right)=13 & \lambda\left(\mathrm{e}_{1,2}\right)=15 & \lambda\left(\mathrm{e}_{1,3}\right)=14 \\ \lambda\left(\mathrm{e}_{2,1}\right)=16 & \lambda\left(\mathrm{e}_{2,2}\right)=18 & \lambda\left(\mathrm{e}_{2,3}\right)=17 \\ \lambda\left(\mathrm{e}_{3,1}\right)=19 & \lambda\left(\mathrm{e}_{3,2}\right)=21 & \lambda\left(\mathrm{e}_{3,3}\right)=20\end{array}$

Setelah diberikan label pada masing-masing titik dan sisi kemudian menjumlahkan semua label titik dan sisi, maka diperoleh :

1. Untuk graf pertama

$\lambda\left(\mathrm{v}_{1,1}\right)+\lambda\left(\mathrm{e}_{1,1}\right)+\lambda\left(\mathrm{v}_{2,1}\right)=2+13+8=23$

$\lambda\left(\mathrm{v}_{2,1}\right)+\lambda\left(\mathrm{e}_{2,1}\right)+\lambda\left(\mathrm{v}_{3,1}\right)=8+16+5=29$

$\lambda\left(\mathrm{v}_{3,1}\right)+\lambda\left(\mathrm{e}_{3,1}\right)+\lambda\left(\mathrm{v}_{4,1}\right)=5+19+11=35$

2. Untuk graf kedua

$\lambda\left(\mathrm{v}_{1,2}\right)+\lambda\left(\mathrm{e}_{1,2}\right)+\lambda\left(\mathrm{v}_{2,2}\right)=3+15+9=27$

$\lambda\left(\mathrm{v}_{2,2}\right)+\lambda\left(\mathrm{e}_{2,2}\right)+\lambda\left(\mathrm{v}_{3,2}\right)=9+18+6=33$ 


$$
\lambda\left(\mathrm{v}_{3,2}\right)+\lambda\left(\mathrm{e}_{3,2}\right)+\lambda\left(\mathrm{v}_{4,2}\right)=6+21+12=27
$$

3. Untuk graf ketiga

$$
\begin{aligned}
& \lambda\left(\mathrm{v}_{1,3}\right)+\lambda\left(\mathrm{e}_{1,3}\right)+\lambda\left(\mathrm{v}_{4,3}\right)=1+14+10=25 \\
& \lambda\left(\mathrm{v}_{2,3}\right)+\lambda\left(\mathrm{e}_{2,3}\right)+\lambda\left(\mathrm{v}_{4,3}\right)=4+17+10=31 \\
& \lambda\left(\mathrm{v}_{3,3}\right)+\lambda\left(\mathrm{e}_{3,3}\right)+\lambda\left(\mathrm{v}_{4,3}\right)=7+20+10=37
\end{aligned}
$$

Dari penjumlahan semua label titik dan sisi pada graf $2 \mathrm{P}_{4} \cup \mathrm{S}_{3,1}$ diatas maka diperoleh suatu $W$ atau himpunan bobot total sisi yang beda dua pada setiap sisinya yaitu $W=\{23,25,27,29,31$, $33,35,37\}$. Jadi, $2 \mathrm{P}_{4} \cup \mathrm{S}_{3,1}$ adalah (23,2)-TSAAS.

\subsubsection{Untuk $n=6 \rightarrow 2 \mathrm{P}_{6} \cup \mathrm{S}_{4,2}$}

Pelabelan TSAAS pada graf $2 \mathrm{P}_{6} \cup \mathrm{S}_{4,2}$, dapat dilakukan seperti pada gambar dibawah ini :

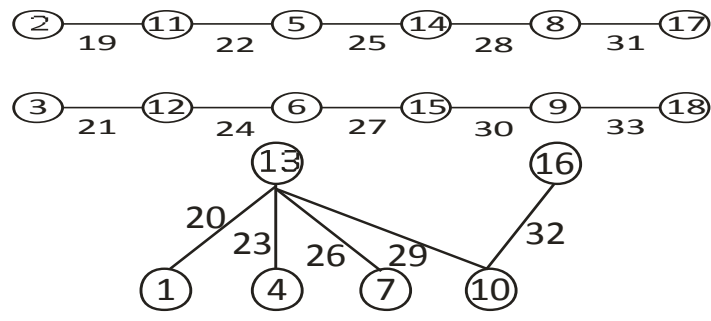

Gambar 3 : Pelabelan Titik dan Sisi Graf $2 \mathrm{P}_{6} \cup \mathrm{S}_{4,2}$

Perhatikan bahwa titik diberi label dengan anggota himpunan $\lambda(\mathrm{V})=\{1,2, \ldots, 18\}$ dan banyaknya titik $n=6$. Jika $\lambda$ adalah pelabelan TSAAS untuk graf $2 \mathrm{P}_{6} \cup \mathrm{S}_{4,2}$ maka diperoleh :

$$
\begin{array}{lll}
\lambda\left(\mathrm{v}_{1,1}\right)=2 & \lambda\left(\mathrm{v}_{1,2}\right)=3 & \lambda\left(\mathrm{v}_{1,3}\right)=1 \\
\lambda\left(\mathrm{v}_{2,1}\right)=11 & \lambda\left(\mathrm{v}_{2,2}\right)=12 & \lambda\left(\mathrm{v}_{2,3}\right)=4 \\
\lambda\left(\mathrm{v}_{3,1}\right)=5 & \lambda\left(\mathrm{v}_{3,2}\right)=6 & \lambda\left(\mathrm{v}_{3,3}\right)=7 \\
\lambda\left(\mathrm{v}_{4,1}\right)=14 & \lambda\left(\mathrm{v}_{4,2}\right)=15 & \lambda\left(\mathrm{v}_{4,3}\right)=10 \\
\lambda\left(\mathrm{v}_{5,1}\right)=8 & \lambda\left(\mathrm{v}_{5,2}\right)=9 & \lambda\left(\mathrm{v}_{5,3}\right)=13 \\
\lambda\left(\mathrm{v}_{6,1}\right)=17 & \lambda\left(\mathrm{v}_{6,2}\right)=18 & \lambda\left(\mathrm{v}_{6,3}\right)=16 \\
\lambda\left(\mathrm{e}_{1,1}\right)=19 & \lambda\left(\mathrm{e}_{1,2}\right)=21 & \lambda\left(\mathrm{e}_{1,3}\right)=20 \\
\lambda\left(\mathrm{e}_{2,1}\right)=22 & \lambda\left(\mathrm{e}_{2,2}\right)=24 & \lambda\left(\mathrm{e}_{2,3}\right)=23 \\
\lambda\left(\mathrm{e}_{3,1}\right)=25 & \lambda\left(\mathrm{e}_{3,2}\right)=27 & \lambda\left(\mathrm{e}_{3,3}\right)=26 \\
\lambda\left(\mathrm{e}_{4,1}\right)=28 & \lambda\left(\mathrm{e}_{4,2}\right)=30 & \lambda\left(\mathrm{e}_{4,3}\right)=29 \\
\lambda\left(\mathrm{e}_{5,1}\right)=31 & \lambda\left(\mathrm{e}_{5,2}\right)=33 & \lambda\left(\mathrm{e}_{5,3}\right)=32
\end{array}
$$

Setelah diberikan label pada masing-masing titik dan sisi kemudian menjumlahkan semua label titik dan sisi, maka diperoleh :

1. Untuk graf pertama

$$
\begin{aligned}
& \lambda\left(\mathrm{v}_{1,1}\right)+\lambda\left(\mathrm{e}_{1,1}\right)+\lambda\left(\mathrm{v}_{2,1}\right)=2+19+11=32 \\
& \lambda\left(\mathrm{v}_{2,1}\right)+\lambda\left(\mathrm{e}_{2,1}\right)+\lambda\left(\mathrm{v}_{3,1}\right)=11+22+5=38 \\
& \lambda\left(\mathrm{v}_{3,1}\right)+\lambda\left(\mathrm{e}_{3,1}\right)+\lambda\left(\mathrm{v}_{4,1}\right)=5+25+14=44
\end{aligned}
$$




$$
\begin{aligned}
& \lambda\left(\mathrm{v}_{4,1}\right)+\lambda\left(\mathrm{e}_{4,1}\right)+\lambda\left(\mathrm{v}_{5,1}\right)=14+28+8=50 \\
& \lambda\left(\mathrm{v}_{5,1}\right)+\lambda\left(\mathrm{e}_{5,1}\right)+\lambda\left(\mathrm{v}_{6,1}\right)=8+31+17=56
\end{aligned}
$$

2. Untuk graf kedua

$$
\begin{aligned}
& \lambda\left(\mathrm{v}_{1,2}\right)+\lambda\left(\mathrm{e}_{1,2}\right)+\lambda\left(\mathrm{v}_{2,2}\right)=3+21+12=36 \\
& \lambda\left(\mathrm{v}_{2,2}\right)+\lambda\left(\mathrm{e}_{2,2}\right)+\lambda\left(\mathrm{v}_{3,2}\right)=12+24+6=42 \\
& \lambda\left(\mathrm{v}_{3,2}\right)+\lambda\left(\mathrm{e}_{3,2}\right)+\lambda\left(\mathrm{v}_{4,2}\right)=6+27+15=48 \\
& \lambda\left(\mathrm{v}_{4,2}\right)+\lambda\left(\mathrm{e}_{4,2}\right)+\lambda\left(\mathrm{v}_{5,2}\right)=15+30+9=54 \\
& \lambda\left(\mathrm{v}_{5,2}\right)+\lambda\left(\mathrm{e}_{5,2}\right)+\lambda\left(\mathrm{v}_{6,2}\right)=9+33+18=60
\end{aligned}
$$

3. Untuk graf ketiga

$$
\begin{aligned}
& \lambda\left(\mathrm{v}_{1,3}\right)+\lambda\left(\mathrm{e}_{1,3}\right)+\lambda\left(\mathrm{v}_{5,3}\right)=1+20+13=34 \\
& \lambda\left(\mathrm{v}_{2,3}\right)+\lambda\left(\mathrm{e}_{2,3}\right)+\lambda\left(\mathrm{v}_{5,3}\right)=4+23+13=40 \\
& \lambda\left(\mathrm{v}_{3,3}\right)+\lambda\left(\mathrm{e}_{3,3}\right)+\lambda\left(\mathrm{v}_{5,3}\right)=7+26+13=46 \\
& \lambda\left(\mathrm{v}_{4,3}\right)+\lambda\left(\mathrm{e}_{4,3}\right)+\lambda\left(\mathrm{v}_{5,3}\right)=10+29+13=52 \\
& \lambda\left(\mathrm{v}_{5,3}\right)+\lambda\left(\mathrm{e}_{5,3}\right)+\lambda\left(\mathrm{v}_{4,3}\right)=16+32+10=58
\end{aligned}
$$

Dari penjumlahan semua label titik dan sisi pada graf $2 \mathrm{P}_{6} \cup \mathrm{S}_{4,2}$ diatas maka diperoleh suatu $W$ atau himpunan bobot total sisi yang beda dua pada setiap sisinya yaitu $W=\{32,34,36,38,40$, 42, 44, 46, 48, 50, 52, 54, 56, 58, 60\}. Jadi, $2 \mathrm{P}_{6} \cup \mathrm{S}_{4,2}$ adalah (32,2)-TSAAS. Berdasarkan beberapa contoh kasus dengan $n$ kecil di atas, maka diperoleh hasil secara umum, untuk graf $2 \mathrm{P}_{n} \cup S_{\frac{n}{2}+1}, \frac{n}{2}-1$ untuk $n \geq 4$ genap dalam teorema berikut ini:

\section{Teorema 1 :}

Graf $2 \mathrm{P}_{n} \cup S_{\frac{n}{2}+1}, \frac{n}{2}-1$ adalah $\left(\frac{9 n}{2}+5,2\right)$-TSAAS untuk $n \geq 4$ genap.

Bukti :

Pandang notasi titik dan sisi pada graf $2 \mathrm{P}_{n} \cup S_{\frac{n}{2}+1}, \frac{n}{2}-1$ dalam persamaan (1). Berikan label pada titik dan sisinya dengan cara :

$$
\begin{gathered}
\lambda\left(v_{i, j}\right)=\left\{\begin{array}{c}
\frac{3 i-1}{2}+1, j=1,1 \leq i \leq n-1 ; i \text { ganjil } \\
\frac{3 n+3 i}{2}-1, j=1,1 \leq i \leq n ; i \text { genap } \\
\frac{3 i-1}{2}+2, j=2,1 \leq i \leq n-1 ; i \text { ganjil } \\
\frac{3 n+3 i}{2}, j=2,1 \leq i \leq n ; i \text { genap } \\
3 i-2, j=3,1 \leq i \leq n
\end{array}\right. \\
\lambda\left(e_{i, j}\right)=\left\{\begin{array}{c}
3 n+3 i-2, j=1,1 \leq i \leq n-1 \\
3 n+3 i, j=2,1 \leq i \leq n-1 \\
3 n+3 i-1, j=3,1 \leq i \leq n-1
\end{array}\right.
\end{gathered}
$$


dengan label tersebut diperoleh :

$a_{i}=\left\{\begin{array}{c}\lambda\left(v_{i, j}\right)+\lambda\left(e_{i, j}\right)+\lambda\left(v_{i, j+i, j}\right), 1 \leq j \leq 2,1 \leq i \leq n-1 \\ \lambda\left(v_{i, 3}\right)+\lambda\left(e_{i, 3}\right)+\lambda\left(v_{\frac{n}{2}+2,3}\right), 1 \leq i \leq \frac{n}{2}+1 \\ \lambda\left(v_{\frac{n}{2}+1,3}\right)+\lambda\left(v_{i, 3}\right)+\lambda\left(v_{i+1,3}\right), \frac{n}{2}+2 \leq i \leq n-1\end{array}\right.$

$a_{i}=\left\{\begin{array}{c}\frac{3 i-1}{2}+1+3 n+3 i-2+\frac{3 n+3 i}{2}-1=\frac{9 n-2}{2}+6 i \\ \frac{3 i-1}{2}+2+3 n+3 i+\frac{3 n+3(i+1)}{2}=\frac{9 n}{2}+6 i+3 \\ 3 i-2+3 n+3 i-1+3\left(\frac{n}{2}+2\right)-2=\frac{9 n}{2}+6 i+1 \\ 3\left(\frac{n}{2}+1\right)-2+3 n+3 i-1+3(i+1)-2=\frac{9 n}{2}+6 i+1\end{array}\right.$

$W=\{a, a+1, a+2, \ldots, a+q-1\}$

$W=\left\{\begin{array}{c}\frac{9 n-2}{2}+6 i \mid 1 \leq i \leq n \\ \frac{9 n}{2}+6 i+3 \mid 1 \leq i \leq n \\ \frac{9 n}{2}+6 i+1 \mid 1 \leq i \leq n\end{array}\right.$

Jadi $\mathrm{W}=\left\{\frac{9 n}{2}+5, \frac{9 n}{2}+7, \frac{9 n}{2}+9, \ldots, \frac{9 n}{2}+n\right\}$, dengan demikian untuk $n \geq 4$ genap dengan graf $2 \mathrm{P}_{n} \mathrm{U}$ $S_{\frac{n}{2}+1,} \frac{n}{2}-1$ mempunyai pelabelan $\left(\frac{9 n}{2}+5,2\right)$-TSAAS.

\subsection{Pelabelan TSAAS Pada Graf $2 P_{n} \cup S_{\frac{n+3}{2}}, \frac{n-3}{2}$ untuk $n \geq 5$ ganjil}

Graf $2 \mathrm{P}_{n} \cup S_{\frac{n+3}{2}}, \frac{n-3}{2}$ untuk $n \geq 5$ ganjil, dapat digambarkan sebagai berikut :

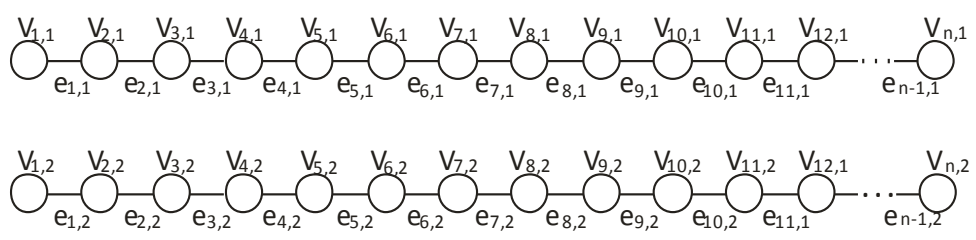




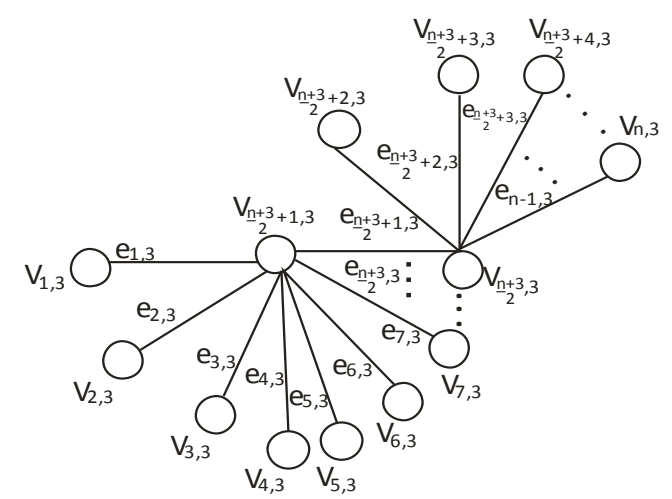

Gambar 4 : Penotasian Titi dan Sisi Graf $2 \mathrm{P}_{n} \cup \frac{n+3}{2}, \frac{n-3}{2}$

Berdasarkan gambar diatas, dapat dinotasikan graf $2 \mathrm{P}_{n} \cup \frac{S_{n+3}}{2}, \frac{n-3}{2}$ dengan himpunan titik dan sisinya sebagai berikut :

$\mathrm{V}\left(2 \mathrm{P}_{n} \cup \frac{\mathrm{S}_{n+3}}{2}, \frac{n}{2}-1\right)=\left\{v_{i, j} \mid 1 \leq j \leq 3,1 \leq i \leq n\right\}$

$\mathrm{E}\left(2 \mathrm{P}_{n} \cup \frac{\mathrm{S}_{n+3}}{2}, \frac{n}{2}-1\right)=\left\{e_{i, j} \mid 1 \leq j \leq 3,1 \leq i \leq n-1\right\}$,

dimana

$e_{i, 1}=v_{i, 1} v_{i+1,1}, 1 \leq i \leq n-1$

$e_{i, 2}=v_{i, 2} v_{i+1,2}, 1 \leq i \leq n-1$

$e_{i, 3}=\left\{\begin{array}{c}v_{i, 3} v_{\frac{n+3}{2}}+1,3,1 \leq i \leq \frac{n}{2}+1 \\ v_{\frac{n+3}{2}, 3} v_{i+1,3}, \frac{n+3}{2}+1 \leq i \leq n-1\end{array}\right.$

3.3. Pelabelan TSAAS Pada Graf $P_{n} \cup 2 S_{\frac{n}{2}+1}, \frac{n}{2}-1$ untuk $n \geq 4$ genap

Graf $\mathrm{P}_{n} \cup 2 \mathrm{~S}_{\frac{n}{2}+1}, \frac{n}{2}-1$ untuk $n \geq 4$ genap, dapat digambarkan sebagai berikut:

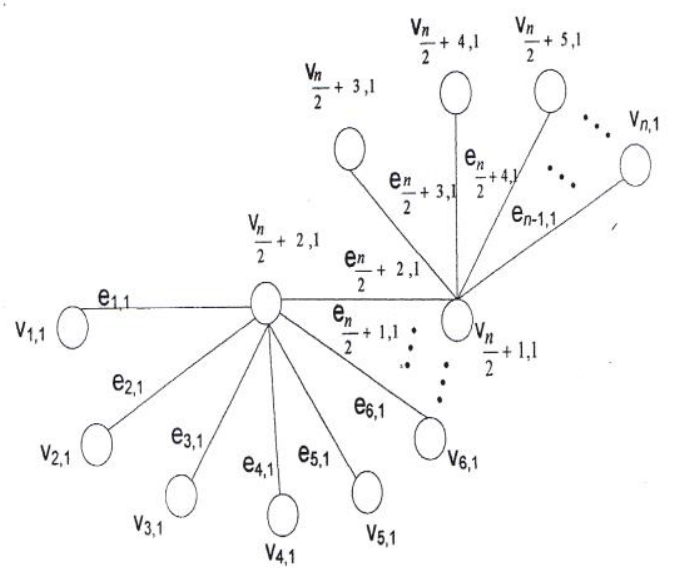




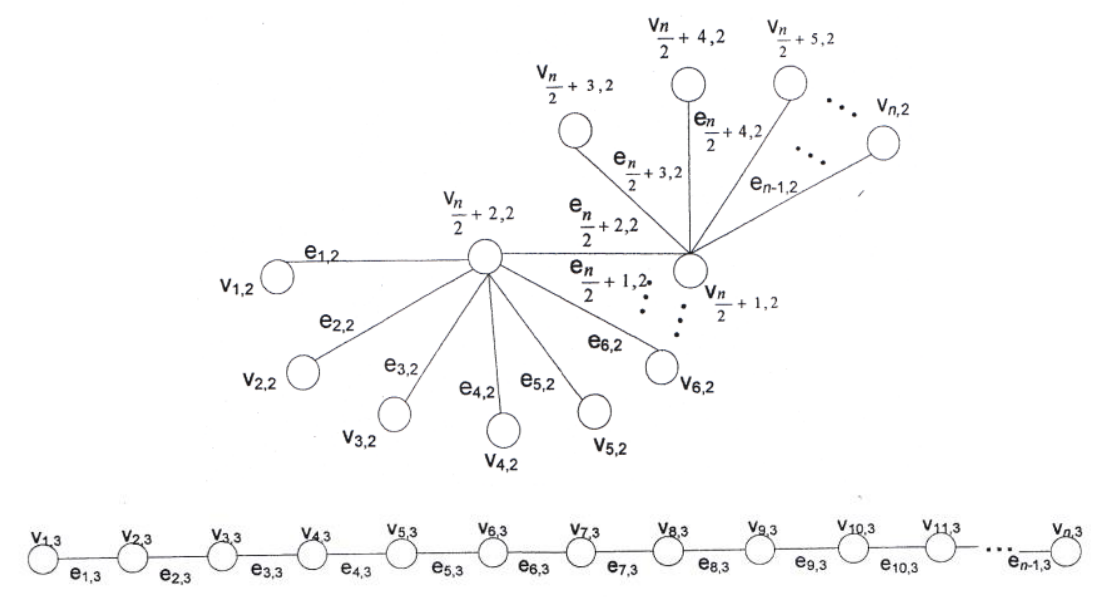

Gambar 5 : Penotasian Titik dan Sisi Graf $P_{n} \cup 2 S_{\frac{n}{2}+1}, \frac{n}{2}-1$

Berdasarkan gambar diatas, dapat di notasikan graf $\mathrm{P}_{n} \cup 2 \mathrm{~S}_{\frac{n}{2}+1}, \frac{n}{2}-1$ dengan himpunan titik dan sisinya sebagai berikut :

$\mathrm{V}\left(\mathrm{P}_{n} \cup 2 \mathrm{~S}_{\frac{n}{2}+1,}, \frac{n}{2}-1\right)=\left\{v_{i, j} \mid 1 \leq j \leq 3,1 \leq i \leq n\right\}$

$\mathrm{E}\left(\mathrm{P}_{n} \cup 2 \mathrm{~S}_{\frac{n}{2}+1}, \frac{n}{2}-1\right)=\left\{e_{i, j} \mid 1 \leq j \leq 3,1 \leq i \leq n-1\right\}$,

dimana

$e_{i, 1}=\left\{\begin{array}{c}v_{i, 1} v_{\frac{n}{2}+2,1}, 1 \leq i \leq \frac{n}{2}+1 \\ v_{\frac{n}{2}+1,1} v_{i+1,1}, \frac{n}{2}+2 \leq i \leq n-1\end{array}\right.$

$e_{i, 2}=\left\{\begin{array}{c}v_{i, 2} v_{\frac{n}{2}+2,2}, 1 \leq i \leq \frac{n}{2}+1 \\ v_{\frac{n}{2}+1,2} v_{i+1,2}, \frac{n}{2}+2 \leq i \leq n-1\end{array}\right.$

$e_{i, 3}=v_{i, 3} v_{i+1,3}, 1 \leq i \leq n-1$

\subsection{Pelabelan TSAAS Pada Graf $P_{n} \cup 2 S_{\frac{n+3}{2}+1}, \frac{n-3}{2}$ untuk $n \geq 5$ ganjil}

Graf $P_{n} \cup 2 S_{\frac{n+3}{2}+1}, \frac{n-3}{2}$ untuk $n \geq 5$ ganjil, dapat dilihat pada Gambar 6 . 


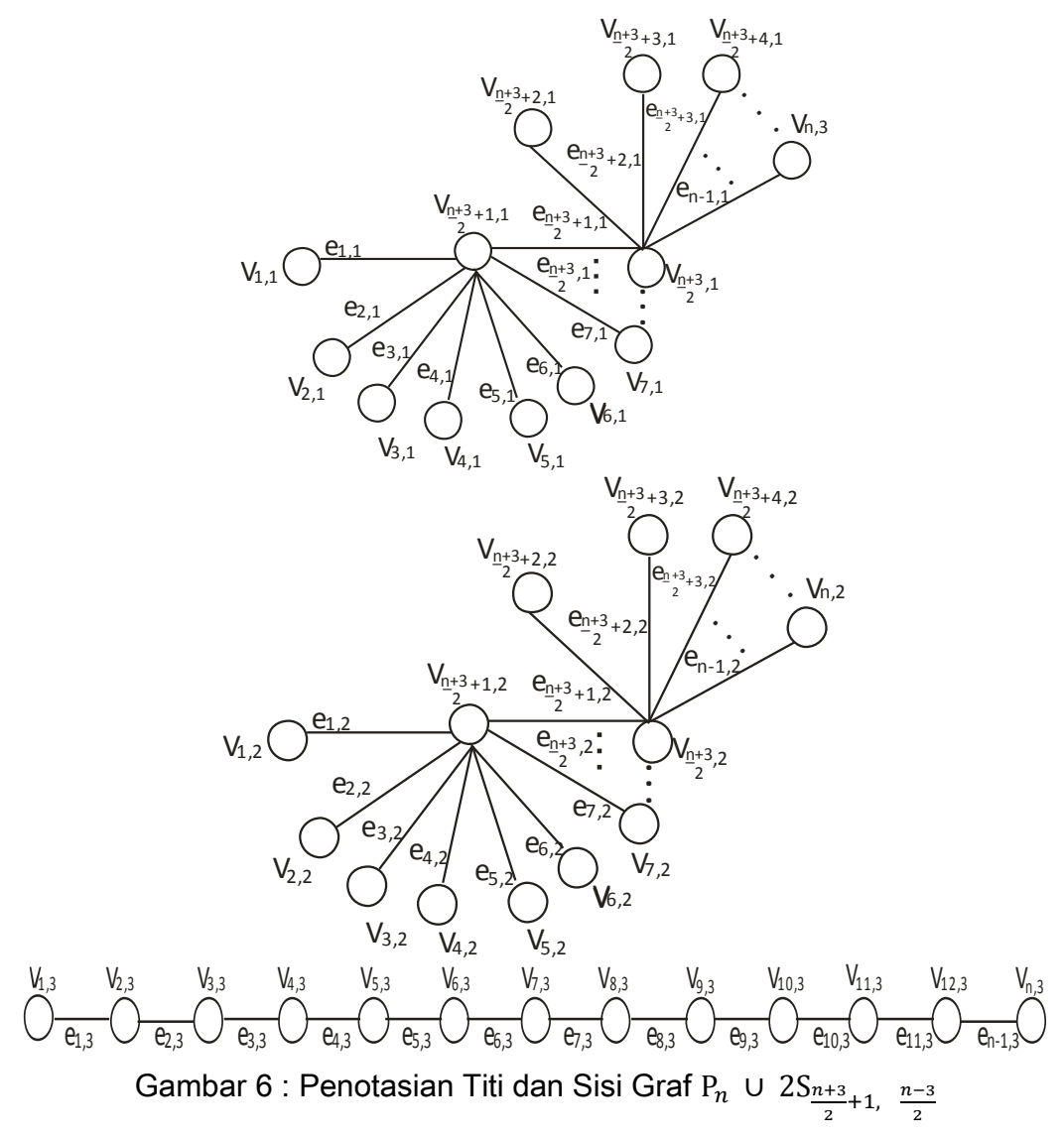

Berdasaran gambar diatas, dapat dinotasikan graf $\mathrm{P}_{n} \cup 2 \mathrm{~S}_{\frac{n+3}{2}+1}, \frac{n-3}{2}$ dengan himpunan titik dan sisi sebagai berikut :

$\mathrm{V}\left(\mathrm{P}_{n} \cup 2 \mathrm{~S}_{\frac{n}{2}+1,} \frac{n}{2}-1\right)=\left\{v_{i, j} \mid 1 \leq j \leq 3,1 \leq i \leq n\right\}$

$\mathrm{E}\left(\mathrm{P}_{n} \cup 2 \mathrm{~S}_{\frac{n}{2}+1,} \frac{n}{2}-1\right)=\left\{e_{i, j} \mid 1 \leq j \leq 3,1 \leq i \leq n-1\right\}$,

dimana

$$
\begin{aligned}
& e_{i, 1}=\left\{\begin{array}{c}
v_{i, 1} \frac{v_{n+3}}{2}+1,1 \\
v_{\frac{n+3}{2}, 1} v_{i+1,1}, \frac{n+3}{2}+1 \leq i \leq n-1
\end{array}\right. \\
& e_{i, 2}=\left\{\begin{array}{c}
v_{i, 2} \frac{v_{n+3}}{2}+1,2 \\
v_{\frac{n+3}{2}, 2} v_{i+1,2}, \frac{n+3}{2}+1 \leq i \leq n-1 \\
e_{i, 3}
\end{array}=v_{i, 3} v_{i+1,3}, 1 \leq i \leq n-1\right.
\end{aligned}
$$




\section{Penutup}

\subsection{Kesimpulan}

Berdasarkan hasil penelitian yang telah dilakukan, dapat disimpulkan bahwa:

1. Graf $2 \mathrm{P}_{n} \cup S_{\frac{n}{2}+1}, \frac{n}{2}-1$ adalah TSAAS dengan $(\mathrm{a}, \mathrm{d})=\left(\frac{9 n}{2}+5,2\right)$ untuk $n \geq 4$ genap. Serta label titik dan sisinya, yaitu :

$$
\begin{aligned}
& \lambda\left(v_{i, j}\right)=\left\{\begin{array}{c}
\frac{3 i-1}{2}+1, j=1,1 \leq i \leq n-1 ; i \text { ganjil } \\
\frac{3 n+3 i}{2}-1, j=1,1 \leq i \leq n ; i \text { genap } \\
\frac{3 i-1}{2}+2, j=2,1 \leq i \leq n-1 ; i \text { ganjil } \\
\frac{3 n+3 i}{2}, j=2,1 \leq i \leq n ; i \text { genap } \\
3 i-2, j=3,1 \leq i \leq n
\end{array}\right. \\
& \lambda\left(e_{i, j}\right)=\left\{\begin{array}{c}
3 n+3 i-2, j=1,1 \leq i \leq n-1 \\
3 n+3 i, j=2,1 \leq i \leq n-1 \\
3 n+3 i-1, j=3,1 \leq i \leq n-1
\end{array}\right.
\end{aligned}
$$

2. Graf $2 \mathrm{P}_{n} \cup S_{\frac{n+3}{2}}, \frac{n-3}{2}$ adalah TSAAS dengan $(\mathrm{a}, \mathrm{d})=\left(\frac{9 n+3}{2}+5,2\right)$ untuk $n \geq 5$ ganjil. Serta label titik dan sisinya, yaitu :

$$
\begin{aligned}
& \lambda\left(v_{i, j}\right)=\left\{\begin{array}{c}
\frac{3 i-1}{2}+1, j=1,1 \leq i \leq n ; i \text { ganjil } \\
\frac{3 n+3 i+1}{2}, j=1,1 \leq i \leq n-1 ; i \text { genap } \\
\frac{3 i-1}{2}+2, j=2,1 \leq i \leq n ; i \text { ganjil } \\
\frac{3 n+3 i+1}{2}+1, j=2,1 \leq i \leq n-1 ; i \text { genap } \\
3 i-2, j=3,1 \leq i \leq n
\end{array}\right. \\
& \lambda\left(e_{i, j}\right)=\left\{\begin{array}{c}
3 n+3 i-2, j=1,1 \leq i \leq n-1 \\
3 n+3 i, j=2,1 \leq i \leq n-1 \\
3 n+3 i-1, j=3,1 \leq i \leq n-1
\end{array}\right.
\end{aligned}
$$

3. Graf $2 \mathrm{P}_{n} \cup S_{\frac{n}{2}+1}, \frac{n}{2}-1$ adalah TSAAS dengan $(\mathrm{a}, \mathrm{d})=\left(\frac{9 n}{2}+6,2\right)$ untuk $n \geq 4$ genap. Serta label titik dan sisinya yaitu : 


$$
\begin{gathered}
\lambda\left(v_{i, j}\right)=\left\{\begin{array}{c}
3 i-2, j=1,1 \leq i \leq n \\
3 i-1, j=2,1 \leq i \leq n \\
\frac{3 i-1}{2}+2, j=3 \quad 1 \leq i \leq n-1 ; i \text { ganjil } \\
\frac{3 n+3 i}{2}, j=3,1 \leq i \leq n ; i \text { genap }
\end{array}\right. \\
\lambda\left(e_{i, j}\right)=\left\{\begin{array}{c}
3 n+3 i-2, j=1,1 \leq i \leq n-1 \\
3 n+3 i, j=2,1 \leq i \leq n-1 \\
3 n+3 i-1, j=3,1 \leq i \leq n-1
\end{array}\right.
\end{gathered}
$$

4. Graf $\mathrm{P}_{n} \cup 2 \mathrm{~S}_{\frac{n+3}{2}+1}, \frac{n-3}{2}$ adalah TSAAS dengan $(\mathrm{a}, \mathrm{d})=\left(\frac{9 n+3}{2}+6,2\right)$ untuk $n \geq 5$ ganjil.

$$
\begin{aligned}
\lambda\left(v_{i, j}\right)=\left\{\begin{array}{r}
3 i-2, j=1, \quad 1 \leq i \leq n \\
3 i-1, j=2, \quad 1 \leq i \leq n \\
\frac{3 i-1}{2}+2, j=3 \quad 1 \leq i \leq n-1 ; i \text { ganjil } \\
\frac{3 n+3 i+1}{2}+1, j=3,1 \leq i \leq n ; i \text { genap }
\end{array}\right. \\
\lambda\left(e_{i, j}\right)=\left\{\begin{array}{l}
3 n+3 i-2, j=1,1 \leq i \leq n-1 \\
3 n+3 i, j=2,1 \leq i \leq n-1 \\
3 n+3 i-1, j=3,1 \leq i \leq n-1
\end{array}\right.
\end{aligned}
$$

\subsection{Saran}

Bagi mahasiswa yang berminat dengn penelitian ini, dapat melakukan penelitian mengenai pelabelan TSAAS pada jenis graf yang lain.

\section{Daftar Pustaka}

[1]. Gallian, J. A., 2011, A Dynamic Survey of Graph Labelling, ElectronicJournal of Combinatorics, Vol. 18, (http://www.emis.ams.org/journal/EJC/Surveys/ds6.pdf), diakses 14 Desember 2011.

[2]. Sri Mei Itasari, Pelabelan Total (a,d) $C_{4}$ anti ajaib super pada graf prisma $C_{n} x P_{2}$. pdf, diakses19 Desember 2011. 